\title{
Differential spatial modulation for high-rate transmission systems
}

\author{
Thu Phuong Nguyen ${ }^{*}$, Xuan Nam Tran ${ }^{1}$, Minh-Tuan Le ${ }^{2}$ and Huan X. Nguyen ${ }^{3}$
}

\begin{abstract}
This paper introduces a new differential spatial modulation (DSM) scheme which subsumes both the previously introduced DSM and high-rate spatial modulation (HR-SM) for wireless multiple input multiple output (MIMO) transmission. By combining the codeword design method of the HR-SM scheme with the encoding method of the DSM scheme, we develop a high-rate differential spatial modulation (HR-DSM) scheme equipped with an arbitrary number of transmit antennas that requires channel state information (CSI) neither at the transmitter nor at the receiver. The proposed approach can be applied to any equal energy signal constellations. The bit error rate (BER) performance of the proposed HR-DSM schemes is evaluated by using both theoretical upper bound and computer simulations. It is shown that for the same spectral efficiency and antenna configuration, the proposed HR-DSM outperforms the DSM in terms of bit error rate (BER) performance.
\end{abstract}

Keywords: MIMO, Differential spatial modulation, High-rate differential spatial modulation

\section{Introduction}

In recent years, many transmission techniques for wireless multiple input multiple output (MIMO) communication systems have been proposed. A byword is the spatial modulation (SM) [1,2], which has attracted increased research interest recently. By exploiting the difference in the channel impulse response of a wireless link from a transmit antenna element to a receive one, the transmit antenna indices are utilized as an additional means to carry information. Compared with the Vertical Bell Laboratories Layered Space-Time (V-BLAST) [3] and space-time block codes (STBCs) $[4,5]$, SM has several advantages which are attained by the following three essential features: (1) unlike the V-BLAST, in an SM scheme, only one transmit antenna is activated during transmission, thereby completely avoiding the problem of inter-channel interference (ICI) among the transmit antennas; (2) since only one antenna is activated for signaling the SM, transmitter needs to use only a single radio frequency (RF) chain, which certainly helps to reduce the hardware cost as well

*Correspondence: phuong.nt@mta.edu.vn

${ }^{1}$ Le Quy Don Technical University, Hoang Quoc Viet Street, Hanoi, Vietnam

Full list of author information is available at the end of the article as energy consumption compared with V-BLAST and STBCs; (3) the spatial position of each transmit antenna is utilized to convey information, thus allowing SM to obtain a spectral efficiency that increases logarithmically with the number of transmit antennas. Coherent SpaceTime Shift Keying (CSTSK) [6-8] is possible of striking a flexible tradeoff between the obtainable diversity and multiplexing gain. This scheme was shown to exhibit a better performance than the SM and SSK schemes since it is possible to obtain both transmit and receive diversity, rather than only receive diversity as in SM. But unlike SM, CSTSK needs multiple RF chains at the transmitter. CSTSK is also inferior to SM in that it requires strict Inter-Antenna Synchronization (IAS) as V-BLAST and STBC. Furthermore, low-complexity ML detection algorithms for SM systems with $M-\mathrm{QAM}, M-\mathrm{PSK}$ modulation are proposed in [9] and [10], respectively. In these detection algorithms, the ML search complexity is independent of the constellation size. As a consequence, SM is a promising MIMO technique over the conventional MIMO schemes.

Until now, most investigations on SM assumed that the CSI is available at the receiver. This assumption is reasonable when the channel varies slowly compared with 
the symbol rate, and knowledge of the channel can be obtained via training. Unfortunately, obtaining channel knowledge is not always realizable when the channel changes quickly. Moreover, for MIMO channels, the number of channel coefficients to be measured is equal to the product of the number of transmit antennas and the number of receive antennas. The length of the training sequence grows proportionally with the number of transmit antennas [11]. This could result in a large decrease of the overall system throughput. In order to dispense with the CSI estimation, a blind detector have been recently proposed for SM [12]. However, this detector requires a large number of observed symbols and thus increased computational complexity for signal estimation. Therefore, solutions that do not require CSI and large observations, such as differential modulation, are very useful.

Differential signaling is a widely used approach to deal with the problem of high-mobility wireless communications without requiring CSI. The differential transmission concept has been successfully implemented in many MIMO systems, e.g., the differential Alamouti scheme [13] and differential spatial multiplexing [14]. However, this approach could hardly be directly applied to SM or Space Shift Keying (SSK) because the channel in SM/SSK systems is the virtual modulation unit, which makes the design of differential SM/SSK unique and difficult. Very recently, a number of differential schemes for SM have been proposed [15-19], called differential SM (DSM), which can be applied to any equal energy signal constellations. Similar to SM, DSM activates only one antenna at a symbol instant. Therefore, ICI is avoided and the requirement of IAS is relaxed. However, the performance of DSM is restricted at a given spectral efficiency.

Motivated by the aforementioned challenge, in this paper, we propose a new DSM scheme based on the concept of spatial constellation (SC), SC codewords [20] and HR-SM codeword [21], called High-Rate Differential Spatial Modulation (HR-DSM), for an arbitrary number of transmit antennas. In the proposed HR-DSM scheme, transmit signal matrix (i.e., HR-DSM codewords) are generated simply by multiplying SC codewords by signal symbols drawn from an $M$-PSK constellation. The role of the SC matrices is to determine how the constellation symbols are weighted and which antenna combination is selected to transmit the HR-DSM codewords. It is noted that the role of SC matrices is similar that of the dispersion matrices (DM) for SM in [7, 8]. However, the designs of these two matrices are based on different approaches. The advantages of the proposed HR-DSM scheme can be elaborated as follows:

- In the proposed HR-DSM scheme, information bits are carried by both SC codewords and constellation symbols. Therefore, a substantial increase in spectral efficiency is achieved as compared to other differential modulation schemes.

- In order to improve the spectral efficiency, it is possible to increase either the signal space $\Omega_{\mathrm{HR}-\mathrm{DSM}}$ or the $M-$ PSK constellation. The scheme is thus more flexible than the DSM [16].

- Compared with the DSM system, the proposed scheme is more advantageous under the space-constraint situations. For the DSM, in order to increase the spectral efficiency, more transmit antennas need to be used. The proposed HR-DSM scheme, however, can keep the number of transmit antenna within the limit and increase the $M$ - PSK constellation size.

- The proposed scheme is superior to the DSM system as it has two separate constellations. In the DSM system, increasing the spectral efficiency reduces distance between the signal points in the DSM constellation. Thanks to having two separate constellations, the proposed HR-DSM can keep the signal space $\Omega_{\mathrm{HR}-\mathrm{DSM}}$ at a required level and increase the $M-$ PSK signal constellation.

The contributions of this paper can be summarized as follows:

- A systematic approach is presented for the design of SC codewords for an arbitrary number of transmit antennas, assuming $n_{T} \geq 2$.

- A theoretical union bound on the bit error rate of the HR-DSM scheme is derived in the closed form. The derived bound can be used as a means to evaluate the BER performance of HR-DSM when the signal-to-noise power ratio (SNR) is sufficiently high.

- Computer simulation results, supported by the theoretical upper bound, are provided to benchmark the BER performance of the propose HR-DSM scheme with those of related differential transmission schemes, such as the differential Alamouti [13], the single-antenna differential scheme (DPSK), the differential scheme of quasi-orthogonal space-time block code (DQOSTBC) [22], and the DSM.

The rest of paper is organized as follows. System model of the proposed HR-DSM is introduced in Section 2. The design of the SC codewords for the HR-DSM is presented in Section 3. In Section 4, a closed-form of pairwise error probability (PEP) and a theoretical upper bound are derived. Section 5 presents simulation results and analysis. Finally, conclusions are drawn in Section 6.

Notation: Throughout the paper, we use the following mathematical notations. $(\cdot)^{H},(\cdot)^{T}$, and $\|\cdot\|_{F}$ denote the Hermitian transpose, transpose, and Frobenius norm of a 
vector or matrix, respectively. $\operatorname{Re}(\cdot)$ denotes the real part of a complex number.

\section{System model}

Figure 1 shows the transmitter and receiver configuration of the proposed HR-DSM with $n_{T}$ transmit antennas and $n_{R}$ receive antennas working over a Rayleigh flatfading channel. Assume that at time instant $t$, symbol $s_{t}$ is transmitted via the $n_{t}$-th transmit antenna, $n_{t}=$ $1,2, \cdots, n_{T}$. The transmitted signal vector is given by $\mathbf{s}=\left[\begin{array}{lllllll}0 & \cdots & 0 & s_{t} & 0 & \cdots & 0\end{array}\right]^{T}$, where only the $n_{t}$-th element is non-zero. In our proposed HR-DSM system, we collect the transmit signal vector $\mathbf{s}$ in time and form a $n_{T} \times T$ actual transmit signal matrix $\mathbf{S}$. As proved in [23], for non-coherent MIMO systems, for arbitrary block length $T$, number of receive antennas $n_{R}$ and signal-tonoise ratio (SNR), the capacities obtained for $n_{T}>T$ and $n_{T}=T$ are equal. Therefore, we set $T=n_{T}$ in our proposed scheme. This means that each transmit signal matrix is sent during $T=n_{T}$ symbol durations. The actual transmit signal matrix $\mathbf{S}$ satisfies the following conditions:
- Only one antenna remains active at each time instant. This means that only one entry in any column of $\mathbf{S}$ is non-zero.

- Each antenna is activated only once in the $n_{T}$ successive time instants of the transmit signal matrix. This means that only one entry in any row of $\mathbf{S}$ is non-zero.

An example of the transmit signal matrix for $n_{T}=3$ is given by $\mathbf{S}=\left[\begin{array}{ccc}0 & s_{12} & 0 \\ s_{21} & 0 & 0 \\ 0 & 0 & s_{33}\end{array}\right]$, where $s_{n_{t} t}$ denotes the transmit symbol over the $n_{t}$-th antenna at time $t$. This matrix determines that at time instant 1 , the symbol $s_{21}$ is transmitted over transmit antenna 2 while transmit antennas 1 and 3 remain idle. Similarly, at time time instants 2 and 3 , the symbols $s_{12}$ and $s_{33}$ are transmitted over antennas 1 and 3, respectively, while the other two transmit antennas remain idle. According to this design, each antenna is activated only once during each block, making differential operation possible so long as the wireless channel remains unchanged over two successive HR-DSM blocks.

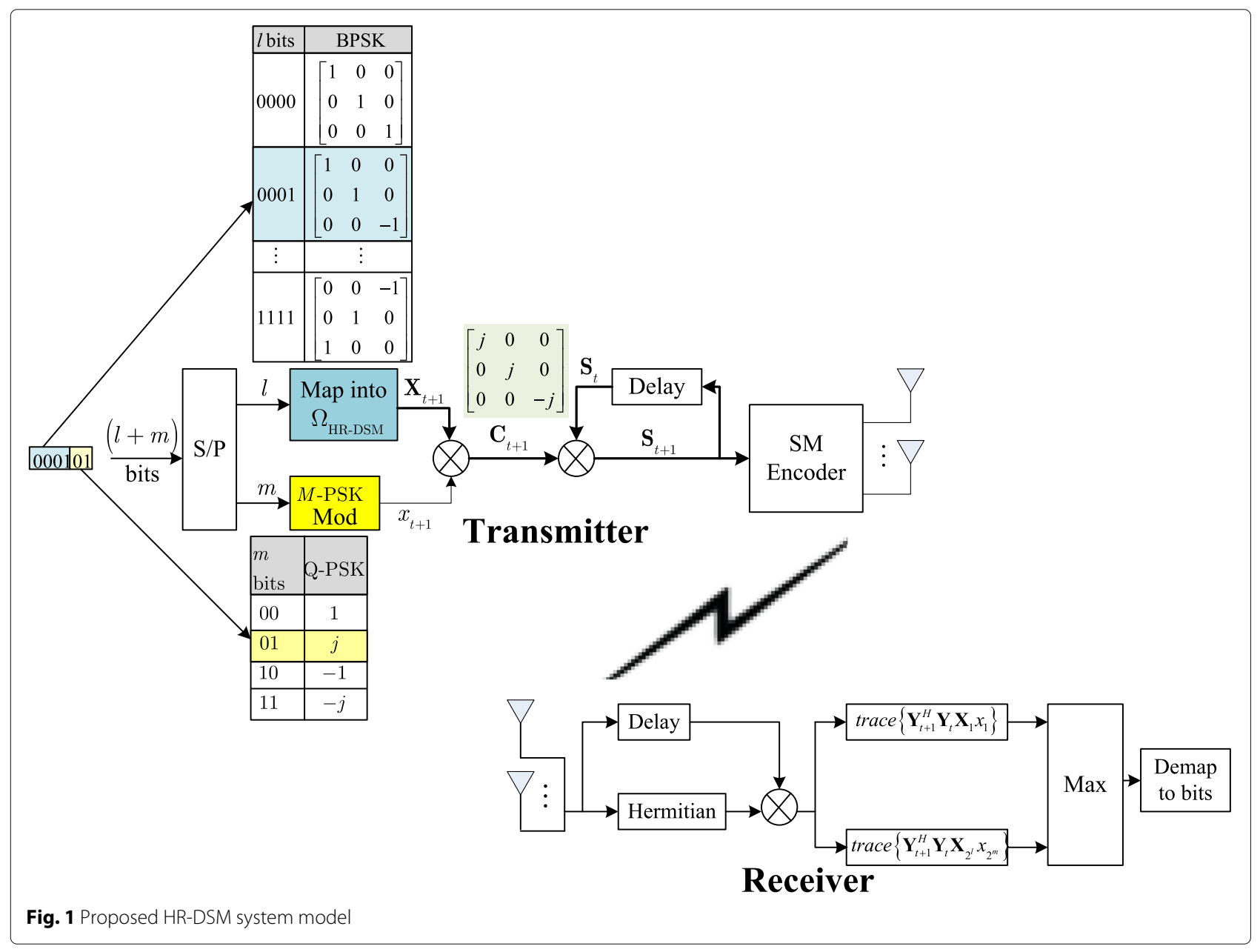


The proposed HR-DSM transmitter works as follows: At time $t+1,(l+m)$ data bits enter the HR-DSM transmitter, among which $l$ bits are mapped into a $n_{T} \times T$ matrix $\mathbf{X}_{t+1}$, out of $K$ matrices in the signal space $\Omega_{H R-D S M}$ with basic elements drawn from $M_{1}$-PSK constellation, while remaining $m=\log _{2} M$ bits are mapped into a $M-$ PSK constellation symbol $x_{t+1}$. The resulting $n_{T} \times T$ HRDSM codeword $\mathbf{C}$ is generated by multiplying $\mathbf{X}$ by $x$, i.e., $\mathbf{C}_{t+1}=\mathbf{X}_{t+1} \cdot x_{t+1}$.

The actual transmitted signal matrix $\mathbf{S}_{t+1}$ is computed via the following formula

$$
\mathbf{S}_{t+1}=\mathbf{S}_{t} \cdot \mathbf{C}_{t+1}
$$

Note that relation Eq. (1) is the fundamental differential transmission relation. Without loss of generality, we choose $\mathbf{C}_{0}=\mathbf{I}_{n_{T}}$, where $\mathbf{I}_{n_{T}}$ is the $n_{T} \times n_{T}$ identity matrix. Then, the chain of transmitted matrices is given by:

$$
\begin{aligned}
& \mathbf{S}_{0}=\mathbf{C}_{0} \\
& \mathbf{S}_{1}=\mathbf{S}_{0} \mathbf{C}_{1}=\mathbf{C}_{0} \mathbf{C}_{1} \\
& \vdots \\
& \mathbf{S}_{t}=\mathbf{S}_{t-1} \mathbf{C}_{t}=\mathbf{C}_{0} \mathbf{C}_{1} \cdots \mathbf{C}_{t}
\end{aligned} .
$$

Let $\mathbf{H}_{t}$ be the $n_{R} \times n_{T}$ fading matrix with the $(i, j)$ th entry $h_{i j}$ denoting the normalized complex fading gain from transmit antenna $j$ to receive antenna $i$. At the receiver side, the chain of received matrices, $\mathbf{Y}_{0}, \ldots, \mathbf{Y}_{t}, \mathbf{Y}_{t+1} \ldots$, is given by

$$
\mathbf{Y}_{t}=\mathbf{H}_{t} \mathbf{S}_{t}+\mathbf{N}_{t}
$$

and

$$
\mathbf{Y}_{t+1}=\mathbf{H}_{t+1} \mathbf{S}_{t+1}+\mathbf{N}_{t+1}
$$

where $\mathbf{Y}_{t}$ is the $n_{R} \times T$ received signal matrix and $\mathbf{N}_{t}$ is the $n_{R} \times T$ AWGN matrix. Using the differential transmission relation in Eqs. (1) and (2), we can rewrite Eq. (3) as

$$
\begin{aligned}
\mathbf{Y}_{t+1} & =\mathbf{H}_{t+1} \mathbf{S}_{t+1}+\mathbf{N}_{t+1} \\
& =\mathbf{H}_{t+1} \mathbf{S}_{t} \mathbf{C}_{t+1}+\mathbf{N}_{t+1} \\
& =\mathbf{Y}_{t} \mathbf{C}_{t+1}-\mathbf{N}_{t} \mathbf{C}_{t+1}+\mathbf{N}_{t+1} \\
& =\mathbf{Y}_{t} \mathbf{C}_{t+1}+\mathbf{N}_{t}{ }^{\prime}
\end{aligned}
$$

where $\mathbf{N}_{t}{ }^{\prime}$ is the $n_{R} \times T$ AWGN matrix. Therefore, to estimate the information matrix, the optimal ML detector can be derived as

$$
\begin{aligned}
\hat{\mathbf{C}}_{t+1} & =\underset{\forall \mathbf{C}}{\arg \min }\left\|\mathbf{Y}_{t+1}-\mathbf{Y}_{t} \mathbf{C}\right\|_{F}^{2} \\
& =\underset{\forall \mathbf{C}}{\arg \min } \operatorname{trace}\left\{\left(\mathbf{Y}_{t+1}-\mathbf{Y}_{t} \mathbf{C}\right)^{H}\left(\mathbf{Y}_{t+1}-\mathbf{Y}_{t} \mathbf{C}\right)\right\} \\
& =\underset{\forall \mathbf{C}}{\arg \max } \operatorname{trace}\left\{\operatorname{Re}\left(\mathbf{Y}_{t+1}^{H} \mathbf{Y}_{t} \mathbf{C}\right)\right\},
\end{aligned}
$$

This is equivalent to

$$
\left\{\hat{\mathbf{X}}_{t+1}, \hat{x}_{t+1}\right\}=\underset{\forall \mathbf{X}, x}{\arg \max } \operatorname{trace}\left\{\operatorname{Re}\left(\mathbf{Y}_{t+1}^{H} \mathbf{Y}_{t} \mathbf{X} x\right)\right\} .
$$

\section{HR-DSM codeword design}

As HR-DSM codewords $\mathbf{C}$ are obtained simply by multiplying matrices $\mathbf{X}$ in the signal space $\Omega_{\mathrm{HR}-\mathrm{DSM}}$ by the constellation symbol $x$, our objective is to design suitable matrix $\mathbf{X}$ for a given $n_{T}$.

For a given $n_{T}$, with $M_{1}$-PSK constellation, we define the following $n_{T} \times 1$ permutation vector $\mathbf{p}_{p}=$ $\left[p_{1} p_{2} \cdots p_{n_{T}}\right],\left(p=1,2, \cdots, n_{T}\right.$ !). For the proposed HR-DSM scheme, we define the basic form of the generator matrix $\mathbf{G}$

$$
\mathbf{G}\left(\mathbf{p}, M_{1}\right)=\frac{1}{\Gamma}\left[\begin{array}{ccc}
x_{11} & \cdots & x_{1 n_{T}} \\
\vdots & \ddots & \vdots \\
x_{n_{T} 1} & \cdots & x_{n_{T} n_{T}}
\end{array}\right]
$$

where column vector $\mathbf{x}_{n}=\left[\begin{array}{llll}x_{1 n} & x_{2 n} & \cdots & x_{n_{T} n}\end{array}\right]^{T},(n=$ $\left.1,2, \cdots, n_{T}\right)$, is a $n_{T} \times 1$ vector with complex valued entries; $\Gamma=\left\|\mathbf{x}_{n}\right\|=\sqrt{\sum_{n_{t}=1}^{n_{T}}\left|x_{n_{t} n}\right|^{2}}$ is the magnitude of $\mathbf{x}$, which is used to normalize the transmit power.

Then, a general procedure for designing the matrix $\mathbf{X}$ for $n_{T}$ transmit antennas is summarized as follows:

1. Assign the first permutation vector to $\mathbf{p}_{1}=\left[\begin{array}{llll}1 & 2 & \cdots & n_{T}\end{array}\right]$.

2. Built $\left(n_{T} !-1\right)$ permutation vectors $\mathbf{p}_{p}$ of $\mathbf{p}_{1}$ : $\mathbf{p}_{2}=\left[\begin{array}{llll}2 & 1 & \cdots & n_{T}\end{array}\right], \cdots, \mathbf{p}_{n_{T} !}=\left[\begin{array}{llll}n_{T} & n_{T-1} & \cdots & 1\end{array}\right]$.

3. Arrange the $\mathbf{G}\left(\mathbf{p}_{p}\right)$ matrices corresponding to permutation vectors $\mathbf{p}_{p}$ as follows:

(a) Fix the $p_{1}$-th element of vector column $\mathbf{x}_{1}$ to 1 and 0 s elsewhere.

(b) Let the $p_{n}$-th elements of remaining column vectors $\mathbf{x}_{n}$ be selected from $M_{1}-$ PSK constellation symbols and 0s elsewhere.

4. Generate the corresponding matrix $\mathbf{X}$ :

$$
\mathbf{X}_{q}=\mathbf{G}\left(\mathbf{p}_{p}, M_{1}\right),\left(q=1,2, \cdots, n_{T} ! M_{1}^{n_{T}-1}\right) .
$$

For example, for $n_{T}=3$, with BPSK constellation, we can construct a signal set $\Omega_{\text {HR-DSM }}$ which includes $q=$ $n_{T} ! M_{1}^{n_{T}-1}=3 ! 2^{2}=24$ matrices $\mathbf{X}$ as follows: 


$$
\begin{aligned}
& \mathbf{X}_{1}=\left[\begin{array}{lll}
1 & 0 & 0 \\
0 & 1 & 0 \\
0 & 0 & 1
\end{array}\right] \quad \mathbf{X}_{2}=\left[\begin{array}{ccc}
1 & 0 & 0 \\
0 & -1 & 0 \\
0 & 0 & 1
\end{array}\right] \\
& \mathbf{X}_{3}=\left[\begin{array}{ccc}
1 & 0 & 0 \\
0 & 1 & 0 \\
0 & 0 & -1
\end{array}\right] \quad \mathbf{X}_{4}=\left[\begin{array}{lll}
1 & 0 & 0 \\
0 & -1 & 0 \\
0 & 0 & -1
\end{array}\right] \\
& \mathbf{X}_{5}=\left[\begin{array}{lll}
1 & 0 & 0 \\
0 & 0 & 1 \\
0 & 1 & 0
\end{array}\right] \quad \mathbf{X}_{6}=\left[\begin{array}{ccc}
1 & 0 & 0 \\
0 & 0 & 1 \\
0 & -1 & 0
\end{array}\right] \\
& \mathbf{X}_{7}=\left[\begin{array}{ccc}
1 & 0 & 0 \\
0 & 0 & -1 \\
0 & 1 & 0
\end{array}\right] \quad \mathbf{X}_{8}=\left[\begin{array}{ccc}
1 & 0 & 0 \\
0 & 0 & -1 \\
0 & -1 & 0
\end{array}\right] \\
& \mathbf{X}_{9}=\left[\begin{array}{lll}
0 & 1 & 0 \\
1 & 0 & 0 \\
0 & 0 & 1
\end{array}\right] \quad \mathbf{X}_{10}=\left[\begin{array}{ccc}
0 & -1 & 0 \\
1 & 0 & 0 \\
0 & 0 & 1
\end{array}\right] \\
& \mathbf{X}_{11}=\left[\begin{array}{ccc}
0 & 1 & 0 \\
1 & 0 & 0 \\
0 & 0 & -1
\end{array}\right] \mathbf{X}_{12}=\left[\begin{array}{ccc}
0 & -1 & 0 \\
1 & 0 & 0 \\
0 & 0 & -1
\end{array}\right] \\
& \mathbf{X}_{13}=\left[\begin{array}{lll}
0 & 0 & 1 \\
1 & 0 & 0 \\
0 & 1 & 0
\end{array}\right] \quad \mathbf{X}_{14}=\left[\begin{array}{ccc}
0 & 0 & 1 \\
1 & 0 & 0 \\
0 & -1 & 0
\end{array}\right] \\
& \mathbf{X}_{15}=\left[\begin{array}{ccc}
0 & 0 & -1 \\
1 & 0 & 0 \\
0 & 1 & 0
\end{array}\right] \mathbf{X}_{16}=\left[\begin{array}{ccc}
0 & 0 & -1 \\
1 & 0 & 0 \\
0 & -1 & 0
\end{array}\right] \\
& \mathbf{X}_{17}=\left[\begin{array}{lll}
0 & 1 & 0 \\
0 & 0 & 1 \\
1 & 0 & 0
\end{array}\right] \quad \mathbf{X}_{18}=\left[\begin{array}{ccc}
0 & -1 & 0 \\
0 & 0 & 1 \\
1 & 0 & 0
\end{array}\right] \\
& \mathbf{X}_{19}=\left[\begin{array}{ccc}
0 & 1 & 0 \\
0 & 0 & -1 \\
1 & 0 & 0
\end{array}\right] \mathbf{X}_{20}=\left[\begin{array}{ccc}
0 & -1 & 0 \\
0 & 0 & -1 \\
1 & 0 & 0
\end{array}\right] \\
& \mathbf{X}_{21}=\left[\begin{array}{lll}
0 & 0 & 1 \\
0 & 1 & 0 \\
1 & 0 & 0
\end{array}\right] \quad \mathbf{X}_{22}=\left[\begin{array}{ccc}
0 & 0 & 1 \\
0 & -1 & 0 \\
1 & 0 & 0
\end{array}\right] \\
& \mathbf{X}_{23}=\left[\begin{array}{ccc}
0 & 0 & -1 \\
0 & 1 & 0 \\
1 & 0 & 0
\end{array}\right] \mathbf{X}_{24}=\left[\begin{array}{ccc}
0 & 0 & -1 \\
0 & -1 & 0 \\
1 & 0 & 0
\end{array}\right]
\end{aligned}
$$

The motivation behind assigning the only non-zero element of vector column $\mathbf{x}_{1}$ to 1 is to guarantee that the proposed HR-DSM scheme obtains high performance. Thanks to this assignment, for the case $\mathbf{X}_{i} \neq \mathbf{X}_{j}$ and $x_{i} \neq x_{j}$, we get $\mathbf{C}_{i} \neq \mathbf{C}_{j}$. Without this assignment, there possibly exist $\mathbf{X}_{i} \neq \mathbf{X}_{j}$ and $x_{i} \neq x_{j}$ such that $\mathbf{C}_{i}=\mathbf{C}_{j}$, leading to a wrong detection at the receiver.

In order for the proposed HR-DSM system to achieve high performance, the transmitted signal space $\Omega_{\mathrm{C}}$ needs to be designed such that the minimum Euclidean distance between two arbitrary transmission signal matrices $\mathbf{C}_{i}$, $\mathbf{C}_{j} \in \Omega_{\mathbf{C}}$ is maximized [24]. Define the difference between two matrices $\mathbf{C}_{i}$ and $\mathbf{C}_{j}$ as $\mathbf{d}_{i, j}=\mathbf{C}_{i}-\mathbf{C}_{j}$, then the Euclidean distance between two transmission signal matrices $\mathbf{C}_{i}$ and $\mathbf{C}_{j}$ can be expressed as

$$
\delta\left(\mathbf{C}_{i}, \mathbf{C}_{j}\right)=\operatorname{det}\left(\mathbf{d}_{i, j}^{H} \mathbf{d}_{i, j}\right) .
$$

Then, for a given spectral efficiency, $M$ and $M_{1}$ are selected such that the following minimum Euclidean distance

$$
\delta_{\min }=\min _{i \neq j} \delta\left(\mathbf{C}_{i}, \mathbf{C}_{j}\right)
$$

is maximized for all pairs of distinct codewords $\mathbf{C}_{i} \neq \mathbf{C}_{j}$ and for all combinations of $\left(M, M_{1}\right)$.

According to the design procedure, for a given $n_{T}$ and with $M_{1}$ - PSK constellation, a total of $Q=$ $n_{T} ! M_{1}^{n_{T}-1}$ matrices $\mathbf{X}$ in the signal space $\Omega_{\mathrm{HR}-\mathrm{DSM}}$ can be obtained. Therefore, one matrix $\mathbf{X}$ is able to carry $l=\left|\log _{2}\left(n_{T} ! M_{1}^{n_{T}-1}\right)\right|$ information bits. In addition, one $M-$ PSK constellation symbol corresponds to $m=\log _{2} M$ information bits. Both of them are transmitted within $n_{T}$ symbol periods. Consequently, the spectral efficiency of the proposed HR-DSM scheme is equal to $\frac{1}{n_{T}}(l+m)=\frac{1}{n_{T}}\left[\left\lfloor\log _{2}\left(n_{T} ! M_{1}^{n_{T}-1}\right)\right\rfloor+\log _{2} M\right]$ bpcu. Clearly, the additional spectral efficiency offered by our proposed scheme is substantially higher than that of DSM.

\section{Theoretical upper bound of HR-DSM}

Suppose that the message $\mathbf{C}_{t}$ is sent at each block. Since errors occur during transmission of the actual transmitted signal matrix $\mathbf{S}_{t}$ due to channel fading and noise, after differential decoding, assume that the message $\mathbf{E}_{t}$ in each block is detected. It follows that $\mathbf{E}_{t} \mathbf{E}_{t}^{H}=\mathbf{I}_{n_{T}}$, where $\mathbf{I}_{n}$ is the $n_{T} \times n_{T}$ identity matrix. In order to measure the difference between $\mathbf{C}_{t}$ and $\mathbf{D}_{t}$, we define $\mathbf{D}_{t}=\mathbf{E}_{t} \mathbf{C}_{t}^{H}$. So the matrix distance between $\mathbf{C}_{t}$ and $\mathbf{E}_{t}$ can be expressed as trace $\left\{\operatorname{Re}\left(\mathbf{I}_{n_{T}}-\mathbf{D}_{t}\right)\right\}$. When no error occurs, $\mathbf{D}_{t}=\mathbf{I}_{n_{T}}$, so trace $\left\{\operatorname{Re}\left(\mathbf{I}_{n_{T}}-\mathbf{D}_{t}\right)\right\}=0$. Since $\mathbf{D}_{t} \mathbf{D}_{t}^{H}=\mathbf{I}_{n_{T}}$, it follows that matrix $\mathbf{D}_{t}$ has the same orthogonal property as the message matrix $\mathbf{C}_{t}$ and the actual transmitted signal $\operatorname{matrix} \mathbf{S}_{t}$.

Recall that the HR-DSM transmitter transmits the matrices $\mathbf{S}_{t}$ and $\mathbf{S}_{t-1}$ instead of directly transmitting the message matrices $\mathbf{C}_{t}$. Due to the influence of fading and noise, suppose that while $\mathbf{S}_{t}$ and $\mathbf{S}_{t-1}$ are transmitted, $\mathbf{Q}_{t}$ and $\mathbf{Q}_{t-1}$ are actually received which causes that the differentially decoded message matrices $\mathbf{C}_{t}$ to become the error message matrices $\mathbf{E}_{t}$. Obviously, $\mathbf{Q}_{t}=\mathbf{E}_{t} \mathbf{Q}_{t-1}=$ $\mathbf{E}_{t} \mathbf{C}_{t-1}$ and $\mathbf{Q}_{t} \mathbf{Q}_{t-1}^{H}=\mathbf{E}_{t}$.

Let $\eta_{c}$ and $\eta_{e}$ be the decision variables for transmission matrices $\mathbf{C}$ and $\mathbf{E}$, respectively. Besides, let $P(\mathbf{C} \rightarrow \mathbf{E} \mid \mathbf{H})$ be the pairwise error probability of deciding $\mathbf{E}$ when $\mathbf{C}$ is transmitted for a given channel realization $\mathbf{H}$. Then, $P(\mathbf{C} \rightarrow \mathbf{E} \mid \mathbf{H})$ can be expressed as

$$
\begin{aligned}
P(\mathbf{C} \rightarrow \mathbf{E} \mid \mathbf{H}) & =P\left(\eta_{e}-\eta_{c}>0 \mid \mathbf{H}\right) \\
& =P\left(\operatorname{trace}\left\{\operatorname{Re}\left(\Phi_{t-1, t}\right)\right\}>0 \mid \mathbf{H}\right),
\end{aligned}
$$


where

$$
\begin{aligned}
& \eta_{c}=\operatorname{trace}\left\{\operatorname{Re}\left(\mathbf{Y}_{t} \mathbf{Y}_{t-1}^{H} \mathbf{S}_{t-1} \mathbf{S}_{t}^{H}\right)\right\}, \\
& \eta_{e}=\operatorname{trace}\left\{\operatorname{Re}\left(\mathbf{Y}_{t} \mathbf{Y}_{t-1}^{H} \mathbf{Q}_{t-1} \mathbf{Q}_{t}^{H}\right)\right\}
\end{aligned}
$$

and

$$
\Phi_{t-1, t}=\eta_{c}-\eta_{e}
$$

Substituting Eq. (2) into Eqs. (10) and (11), we have

$$
\begin{aligned}
& \operatorname{trace}\left\{\operatorname{Re}\left(\Phi_{t-1, t}\right)\right\} \simeq \operatorname{trace}\left\{\operatorname{Re}\left(\mathbf{H}_{t-1} \mathbf{H}_{t}^{H}\left(\mathbf{D}_{t}-\mathbf{I}_{n_{T}}\right)\right)\right\} \\
& +\operatorname{trace}\left\{\operatorname { R e } \left(\mathbf{S}_{t} \mathbf{H}_{t} \mathbf{N}_{t-1}^{H} \mathbf{Q}_{t-1} \mathbf{Q}_{t}^{H}+\mathbf{N}_{t} \mathbf{H}_{t-1}^{H} \mathbf{C}_{t-1}^{H} \mathbf{Q}_{t-1} \mathbf{Q}_{t}^{H}\right.\right. \\
& \left.\left.-\mathbf{S}_{t} \mathbf{H}_{t} \mathbf{N}_{t-1}^{H} \mathbf{S}_{t-1} \mathbf{S}_{t}^{H}-\mathbf{N}_{t} \mathbf{H}_{t-1}^{H} \mathbf{S}_{t-1}^{H} \mathbf{S}_{t-1} \mathbf{S}_{t}^{H}\right)\right\} .
\end{aligned}
$$

Note that the second-order noise terms in Eq. (12) are ignored since they are quite small compared to other noise terms when SNR is large enough. Let

$$
\begin{aligned}
\Delta & =\operatorname{trace}\left\{\operatorname{Re}\left(\Phi_{t-1, t}\right)\right\} \\
& =-\left(\sum_{\substack{i=1, \ldots, n_{R} \\
j=1, \ldots, n_{T}}}\left|h_{i j}\right|^{2}\right) \rho+\operatorname{trace}\{\operatorname{Re}(\Theta)\},
\end{aligned}
$$

where $\rho$ is defined as

$$
\rho=\operatorname{trace}\left\{\operatorname{Re}\left(\mathbf{I}_{n_{T}}-\mathbf{D}_{t}\right)\right\},
$$

and

$$
\begin{aligned}
\Theta= & \mathbf{S}_{t} \mathbf{H}_{t} \mathbf{N}_{t-1}^{H} \mathbf{Q}_{t-1} \mathbf{Q}_{t}^{H}+\mathbf{N}_{t} \mathbf{H}_{t-1}^{H} \mathbf{C}_{t-1}^{H} \mathbf{Q}_{t-1} \mathbf{Q}_{t}^{H} \\
& -\mathbf{S}_{t} \mathbf{H}_{t} \mathbf{N}_{t-1}^{H} \mathbf{S}_{t-1} \mathbf{S}_{t}^{H}-\mathbf{N}_{t} \mathbf{H}_{t-1}^{H} \mathbf{S}_{t-1}^{H} \mathbf{S}_{t-1} \mathbf{S}_{t}^{H}
\end{aligned}
$$

For given transmission matrices $\mathbf{C}$ and $\mathbf{E}, \mathbf{Q}_{t-1}, \mathbf{Q}_{t}, \mathbf{S}_{t-1}$ and $\mathbf{S}_{t}$, and $\rho$ can be considered as deterministic quantities. Therefore, we can easily show that $E[\operatorname{trace}\{\operatorname{Re}(\Theta)\}]=0$. Taking the expectation of both sides of Eq. (13), we have:

$$
\begin{aligned}
E[\Delta] & =-\left(\sum_{\substack{i=1, \ldots, n_{R} \\
j=1, \ldots, n_{T}}}\left|h_{i j}\right|^{2}\right) E[\rho]+E[\operatorname{trace}\{\operatorname{Re}(\Theta)\}] \\
& =-\left(\sum_{\substack{i=1, \ldots, n_{R} \\
j=1, \ldots, n_{T}}}\left|h_{i j}\right|^{2}\right) \rho .
\end{aligned}
$$

The computation of the variance of $\Delta$ is more complicated, because some terms in Eq. (15) are correlated, although most of the terms are assumed to be mutually independent. It is proved in the "Appendix" section that the variance of $\Delta$ is given by:

$$
\begin{aligned}
& \operatorname{Var}[\Delta] \\
& =\operatorname{Var}[\operatorname{trace}\{\operatorname{Re}(\Theta)\}] \\
& =8\left(\sum_{\substack{i=1, \ldots, n_{R} \\
j=1, \ldots, n_{T}}}\left|h_{i j}\right|^{2}\right) N_{0} \\
& -4 N_{0}\left(\sum_{\substack{i=1, \ldots, n_{R} \\
j=1, \ldots, n_{T}}}\left|h_{i j}\right|^{2}\right) \operatorname{trace}\left\{\operatorname{Re}\left(\mathbf{D}_{t}\right)\right\}
\end{aligned}
$$

which can be simplified to

$$
\operatorname{Var}[\Delta]=2 \rho\left(\sum_{\substack{i=1, \ldots, n_{R} \\ j=1, \ldots, n_{T}}}\left|h_{i j}\right|^{2}\right) N_{0}
$$

From Eqs. (9), (13), (16), and (18), it follows that

$$
\begin{aligned}
& \operatorname{Pr}(\mathbf{C} \rightarrow \mathbf{E} \mid \mathbf{H}) \\
& =\operatorname{Pr}\left(\eta_{e}-\eta_{c}>0 \mid \mathbf{H}\right) \\
& =\operatorname{Pr}(\Delta>0 \mid \mathbf{H}) \\
& =Q\left(\sqrt{\gamma \rho\left(\sum_{\substack{i=1, \ldots, n_{R} \\
j=1, \ldots, n_{T}}}\left|h_{i j}\right|^{2}\right) / 4}\right),
\end{aligned}
$$

where $Q$ denotes the Gaussian tail function, and $\gamma=$ $E_{s} / N_{0}$ is the SNR per symbol. Defining the instantaneous SNR as

$$
\gamma_{b}=\gamma\left(\sum_{\substack{i=1, \ldots, n_{R} \\ j=1, \ldots, n_{T}}}\left|h_{i j}\right|^{2}\right)
$$

and using the alternative form of the Gaussian Q-function [25], we can write

$$
\begin{aligned}
\operatorname{Pr}(\mathbf{C} \rightarrow \mathbf{E} \mid \mathbf{H}) & =Q\left(\sqrt{(\rho / 2) \gamma_{b}}\right) \\
& =\frac{1}{\pi} \int_{0}^{\pi / 2} \exp \left(-\frac{(\rho / 2) \gamma_{b}}{2 \sin ^{2} \theta}\right) d \theta .
\end{aligned}
$$

Averaging Eq. (21) over all realizations of the channel matrix $\mathbf{H}$, we obtain the PEP as

$$
\operatorname{Pr}(\mathbf{C} \rightarrow \mathbf{E})=\int_{0}^{\infty} Q\left(\sqrt{(\rho / 2) \gamma_{b}}\right) p\left(\gamma_{b}\right) d \gamma_{b} .
$$

where $p\left(\gamma_{b}\right)$ is the PDF of $\gamma_{b}$ given in [26].

Let $\mathbf{u}$ represent a sequence with $q$ information bits and $\hat{\mathbf{u}}$ denotes an error sequence with the same number 
of information bits. The bit error probability $P_{b}$ of the proposed HR-DSM scheme is union-bounded by [27, 28]:

$$
P_{b} \leq \frac{1}{2 q} \sum_{\mathbf{C} \neq \mathbf{E}} \operatorname{Pr}(\mathbf{C} \rightarrow \mathbf{E}) \cdot w(\mathbf{u}, \hat{\mathbf{u}}),
$$

where $w(\mathbf{u}, \hat{\mathbf{u}})$ is the Hamming distance between sequences $\mathbf{u}$ and $\hat{\mathbf{u}}$. The $\operatorname{PEP} \operatorname{Pr}(\mathbf{C} \rightarrow \mathbf{E})$ is given by Eq. (22).

\section{Performance evaluation}

In this section, Monte Carlo simulations and the theoretical upper bound are used to study the BER performance of the proposed HR-DSM scheme for different antenna arrangements, as well as to compare them against different MIMO systems, such as coherent detection SM [1] and DSM [15], the differential scheme of the well known Alamouti scheme [13], the DPSK scheme, DQOSTBC scheme [22]. Simulations are carried out over the quasistatic Rayleigh fading channel. We assume that the channel state information is perfectly known at the receiver of an SM system. In addition, ML detection is applied to all systems under consideration.

\subsection{Comparison between theoretical and simulation results}

In Fig. 2, the theoretical and simulation BER performances versus the signal-to-noise power ratio (SNR) are plotted. The theoretical and simulation results match well in the high SNR regions for all cases. This implies that the bound given by Eq. (23) can be used as a tool to evaluate BER performances of not only the proposed HR-DSM scheme but also the DSM scheme when SNR is sufficiently high.

\subsection{Comparison between the differential detection HR-DSM and the coherent detection SM}

In order to support our analysis in Section 4, Fig. 3 presents an example to compare the differential detection scheme of HR-DSM with the coherent-detection SM scheme. The simulations are realized with $n_{R}=4$ and $n_{T}=2$, 4. The BER performances of the differential and the coherent schemes are compared under the same spectral efficiency of 3 bpcu. Note that when SM and HR-DSM use the same signal constellation, their spectral efficiencies are not equal.

It is observed from the figure that compared to the SM, HR-DSM suffers from a performance degradation of less than $3 \mathrm{~dB}$ in the SNR, particularly in the high SNR region.

\subsection{BER performance comparison of proposed HR-DSM and other transmission schemes.}

In Fig. 4, we compare the BER performance of HR-DSM and DSM with the same spectral efficiency of $2.5 \mathrm{bpcu}$. To obtain spectral efficiency of $2.5 \mathrm{bpcu}$, the HR-DSM utilizes BPSK and 8-PSK constellations, while DSM adopts the QPSK constellation. The simulations are carried out with $n_{T}=2$ and $n_{R}=1,2$, and 4 . It can be seen from the figure that the proposed HR-DSM outperforms DSM.

Figure 5 shows the BER curves of HR-DSM and other transmission schemes with the spectral efficiency of 3 bpcu. The first one is the differential scheme of the wellknown Alamouti scheme (DA) with $n_{T}=2$ [13]. The

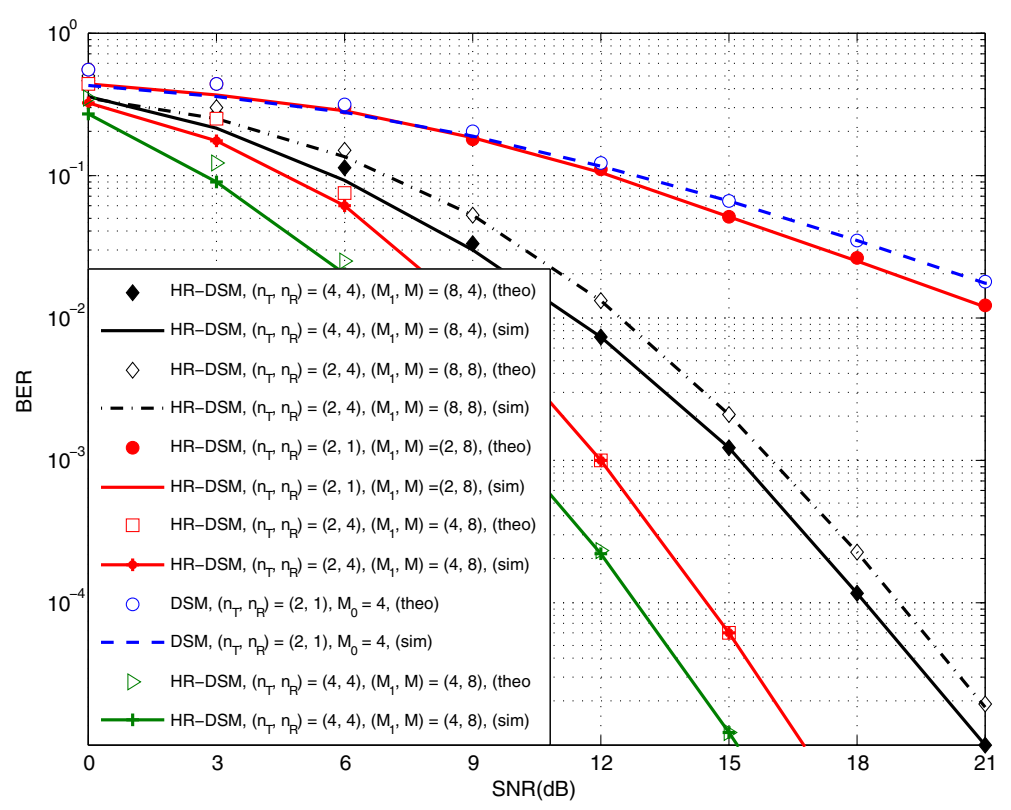

Fig. 2 Theoretical and simulation results of BER performances of HR-DSM 


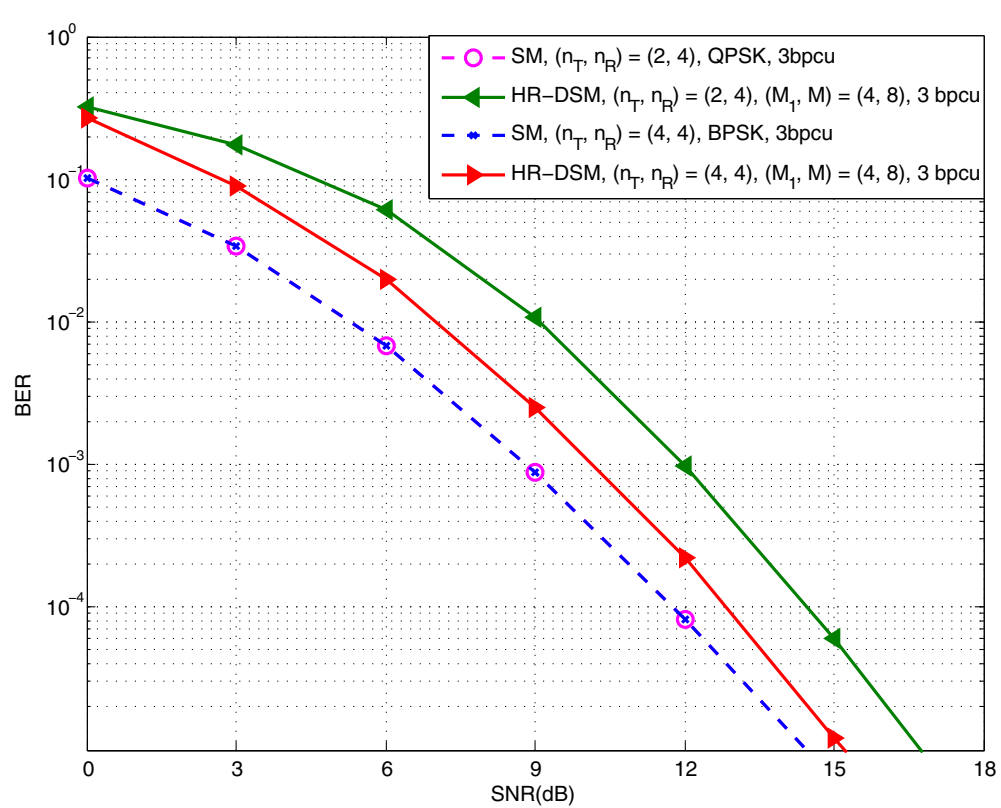

Fig. 3 BER performance comparison of the differential detection HR-DSM and the coherent detection SM

second is the single-antenna differential scheme, DPSK. The third is the differential scheme of quasi-orthogonal space-time block code [22] (DQOSTBC) with $n_{T}=4$. And the last is the DSM. Simulation results indicate that, when $n_{R}=1,2$, the proposed HR-DSM exhibits the worse performance than DA and DQOSTBC. However, when $n_{R}=4$, HR-DSM outperforms all the remaining schemes. This is because when $n_{R}=1,2$, the erroneous antenna detection of HR-DSM is so significant that it redeems its coding gain. Increase in $n_{R}$ reduces the erroneous antenna detection of HR-DSM considerably. Therefore, when $n_{R}=4$, the coding gain of HR-DSM due to the use of lower order modulation dominates the result and thus HR-DSM outperforms the others.

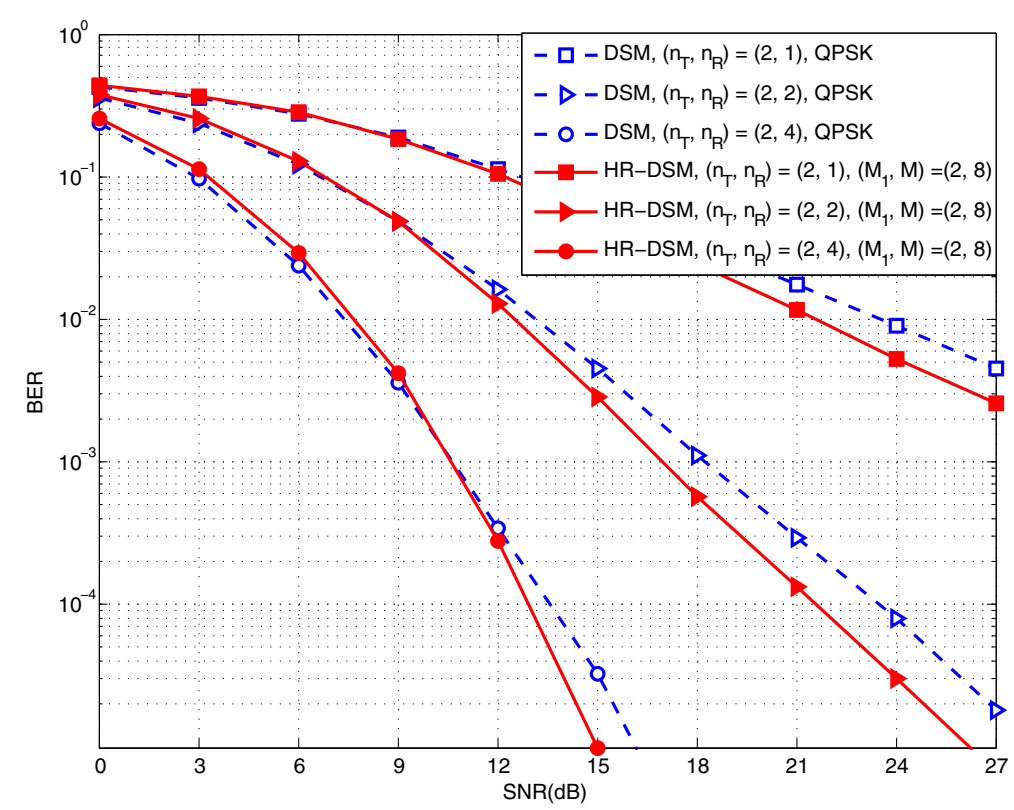

Fig. 4 BER performance comparison of proposed HR-DSM and DSM at 2.5 bpcu $\left(n_{T}=2\right)$ 


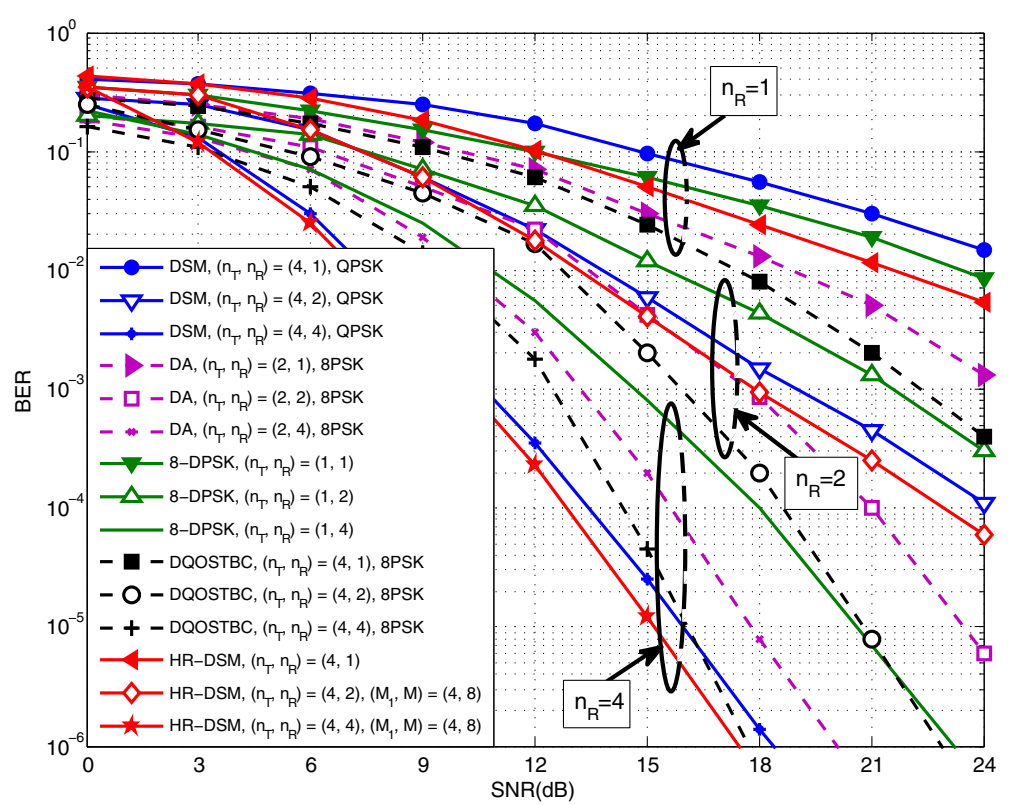

Fig. 5 BER performance comparison of proposed HR-DSM and other transmission schemes at $3 \mathrm{bpcu}$

In addition, it can be observed that in all antenna configurations, DA and DQOSTBC benefit more from increasing the SNR than does DSM and HR-DSM. This is because the diversity order of HR-DSM, as well as DSM, approaches that of single antenna system which is $n_{R}$ as detailed in [2]. In contrast, the Alamouti scheme with $n_{T}=2$ provides full diversity, i.e., $2 n_{R}$ and QOSTBC with $n_{T}=4$ provides half of the maximum achievable diversity. Thus, at high SNR, DA and DQOSTBC outperform HR-DSM and DSM.

\subsection{HR-DSM with multiple transmit antennas}

Figure 6 compares the BER performance of HR-DSM with multiple transmit antennas with the same spectral efficiency of $3 \mathrm{bpcu}$. To obtain spectral efficiency of 3 bpcu, all antenna configurations utilize QPSK and 8-PSK



Fig. 6 Simulation results of the HR-DSM for 2, 3, and 4 transmit antennas 
constellations, i.e., $\left(M_{1}, M\right)=(4,8)$. It can be seen from the figure that although all antenna configurations utilize the same modulation order, the HR-DSM system with $n_{T}=4$ provides the best BER performance, the HR-DSM system with $n_{T}=3$ has better BER performance compared with the HR-DSM system having $n_{T}=2$. In other words, the more transmit antennas are used, the less the performance loss of HR-DSM is as compared to SM. This is because in the configuration with $n_{T}=3,4$, the best set of the signal space $\Omega_{\text {HR-DSM }}$ is selected in order to obtain improved performance.

Another observation is that for the same number of receive antennas, HR-DSM configurations with $n_{R}=1$ will provide almost same performance. For $n_{R}=2$, 4, HR-DSM with $n_{T}=4$ outperforms HR-DSM with $n_{T}=2$, 3. The likelihood of erroneous antenna detection decreases with an increasing number of receive antennas. This observation can be used as a guideline for selecting the signal constellation and the spatial configuration. To get the full potential of HR-DSM, the system should be equipped with sufficient receive antennas.

\section{Conclusions}

In this paper, a new differential space time modulation scheme based on the HR-SM codeword is proposed for MIMO systems. In addition, a theoretical upper bound is derived for the evaluation of the BER of the proposed HRDSM scheme. Simulation results show that this scheme outperforms the DSM scheme in terms of BER performance. The performance of the proposed scheme can be improved by selecting the set of transmission matrices having the largest minimum Euclidean distance. Undoubtedly, the proposed method paves a new way to design differential SM schemes with more transmit antennas.

\section{Appendix}

\section{Evaluation of the variance of trace $\{\operatorname{Re}(\Theta)\}$}

First, we can write

$$
\operatorname{trace}\{\operatorname{Re}(\Theta)\}=\operatorname{trace}\left\{\operatorname{Re}\left(\Theta_{1}\right)\right\}-\operatorname{trace}\left\{\operatorname{Re}\left(\Theta_{2}\right)\right\} \text {, }
$$

where

$$
\begin{aligned}
& \Theta_{1}=\mathbf{S}_{t} \mathbf{H}_{t} \mathbf{N}_{t-1}^{H} \mathbf{Q}_{t-1} \mathbf{Q}_{t}^{H}+\mathbf{N}_{t} \mathbf{H}_{t-1}^{H} \mathbf{C}_{t-1}^{H} \mathbf{Q}_{t-1} \mathbf{Q}_{t}^{H}, \\
& \Theta_{2}=\mathbf{S}_{t} \mathbf{H}_{t} \mathbf{N}_{t-1}^{H} \mathbf{S}_{t-1} \mathbf{S}_{t}^{H}+\mathbf{N}_{t} \mathbf{H}_{t-1}^{H} \mathbf{S}_{t-1}^{H} \mathbf{S}_{t-1} \mathbf{S}_{t}^{H},
\end{aligned}
$$

Now, the variance of trace $\{\operatorname{Re}(\Theta)\}$ is given by:

$$
\begin{aligned}
& \operatorname{Var}[\operatorname{trace}\{\operatorname{Re}(\Theta)\}] \\
& \quad=\operatorname{Var}\left[\operatorname{trace}\left\{\operatorname{Re}\left(\Theta_{1}\right)\right\}\right]+\operatorname{Var}\left[\operatorname{trace}\left\{\operatorname{Re}\left(\Theta_{2}\right)\right\}\right] \\
& -2 \operatorname{Cov}\left(\operatorname{trace}\left\{\operatorname{Re}\left(\Theta_{1}\right)\right\}, \operatorname{trace}\left\{\operatorname{Re}\left(\Theta_{2}\right)\right\}\right) .
\end{aligned}
$$

From Eq. (26), we can see that the variance of trace $\{\operatorname{Re}(\Theta)\}$ consists of three parts: the variance of trace $\left\{\operatorname{Re}\left(\Theta_{1}\right)\right\}$, the variance of trace $\left\{\operatorname{Re}\left(\Theta_{2}\right)\right\}$, and the cross-correlation between trace $\left\{\operatorname{Re}\left(\Theta_{1}\right)\right\}$ and trace $\left\{\operatorname{Re}\left(\Theta_{2}\right)\right\}$. These quantities will be evaluated in the following sub-sections.

Evaluation of $\operatorname{Var}\left[\operatorname{trace}\left\{\operatorname{Re}\left(\Theta_{1}\right)\right\}\right], \operatorname{Var}\left[\operatorname{trace}\left\{\operatorname{Re}\left(\Theta_{2}\right)\right\}\right]$ trace $\left\{\operatorname{Re}\left(\Theta_{1}\right)\right\}$ can be evaluated as follows:

$$
\begin{aligned}
\operatorname{Var} & {\left[\operatorname{trace}\left\{\operatorname{Re}\left(\Theta_{1}\right)\right\}\right] } \\
= & \operatorname{Var}\left[\operatorname{trace}\left\{\operatorname{Re}\left(\mathbf{Q}_{t-1} \mathbf{Q}_{t}^{H} \mathbf{S}_{t} \mathbf{H}_{t} \mathbf{N}_{t-1}^{H}\right)\right\}\right] \\
& +\operatorname{Var}\left[\operatorname{trace}\left\{\operatorname{Re}\left(\mathbf{Q}_{t-1} \mathbf{Q}_{t}^{H} \mathbf{S}_{t-1} \mathbf{H}_{t-1} \mathbf{N}_{t}^{H}\right)\right\}\right] \\
= & \left.4 \sum_{\substack{i=1, \ldots, n_{R} \\
j=1, \ldots, n_{T}}}\left|h_{i j}\right|^{2}\right) N_{0} .
\end{aligned}
$$

It could be observed from Eq. (25) that trace $\left\{\operatorname{Re}\left(\Theta_{1}\right)\right\}$ and trace $\left\{\operatorname{Re}\left(\Theta_{2}\right)\right\}$ have the same structure. Therefore, we can similarly get:

$$
\operatorname{Var}\left[\operatorname{trace}\left\{\operatorname{Re}\left(\Theta_{2}\right)\right\}\right]=4\left(\sum_{\substack{i=1, \ldots, n_{R} \\ j=1, \ldots, n_{T}}}\left|h_{i j}\right|^{2}\right) N_{0} .
$$

Finally, we can write

$$
\begin{aligned}
\operatorname{Var} & {\left[\operatorname{trace}\left\{\operatorname{Re}\left(\Theta_{1}\right)\right\}\right]+\operatorname{Var}\left[\operatorname{trace}\left\{\operatorname{Re}\left(\Theta_{2}\right)\right\}\right] } \\
& =8\left(\sum_{\substack{i=1, \ldots, n_{R} \\
j=1, \ldots, n_{T}}}\left|h_{i j}\right|^{2}\right) N_{0} .
\end{aligned}
$$

\section{Evaluation of $\operatorname{Cov}\left(\operatorname{trace}\left\{\operatorname{Re}\left(\Theta_{1}\right)\right\}, \operatorname{trace}\left\{\operatorname{Re}\left(\Theta_{2}\right)\right\}\right)$}

The cross-correlation $\operatorname{Cov}\left(\operatorname{trace}\left\{\operatorname{Re}\left(\Theta_{1}\right)\right\}\right.$, trace $\left.\left\{\operatorname{Re}\left(\Theta_{2}\right)\right\}\right)$ can be expressed as

$$
\begin{aligned}
\Psi & =\operatorname{Cov}\left(\operatorname{trace}\left\{\operatorname{Re}\left(\Theta_{1}\right)\right\}, \operatorname{trace}\left\{\operatorname{Re}\left(\Theta_{2}\right)\right\}\right) \\
& =\operatorname{Cov}\left(\delta_{1}, \delta_{2}\right) \\
& =2 N_{0}\left(\sum_{\substack{i=1, \ldots, n_{R} \\
j=1, \ldots, n_{T}}}\left|h_{i j}\right|^{2}\right) \times \operatorname{trace}\left\{\operatorname{Re}\left(\mathbf{D}_{t}\right)\right\},
\end{aligned}
$$

where

$$
\begin{aligned}
\delta_{1}=\operatorname{trace} & \left\{\operatorname { R e } \left(\mathbf{S}_{t} \mathbf{H}_{t} \mathbf{N}_{t-1}^{H} \mathbf{Q}_{t-1} \mathbf{Q}_{t}^{H}\right.\right. \\
+ & \left.\left.\mathbf{N}_{t} \mathbf{H}_{t-1}^{H} \mathbf{S}_{t-1}^{H} \mathbf{Q}_{t-1} \mathbf{Q}_{t}^{H}\right)\right\},
\end{aligned}
$$

and

$$
\begin{aligned}
\delta_{2} & =\operatorname{trace}\left\{\operatorname{Re}\left(\mathbf{S}_{t} \mathbf{H}_{t} \mathbf{N}_{t-1}^{H} \mathbf{S}_{t-1} \mathbf{S}_{t}^{H}+\mathbf{N}_{t} \mathbf{H}_{t-1}^{H} \mathbf{S}_{t-1}^{H} \mathbf{S}_{t-1} \mathbf{S}_{t}^{H}\right)\right\} \\
& =\operatorname{trace}\left\{\operatorname{Re}\left(\mathbf{H}_{t} \mathbf{N}_{t-1}^{H} \mathbf{S}_{t-1}+\mathbf{N}_{t} \mathbf{H}_{t-1}^{H} \mathbf{S}_{t}^{H}\right)\right\} .
\end{aligned}
$$

Proof Since $\mathbf{S}_{t}, \mathbf{H}_{t}$ and $\mathbf{Q}_{t}$ are matrices with deterministic entries, the cross-correlation $\Psi$ is related to the 2 noise matrices $\mathbf{N}_{t}, \mathbf{N}_{t-1}$ only. Moreover, these noise matrices 
are mutually independent of each other, and any crosscorrelation between two terms with different noise matrices is zero. Hence $\Psi$ only consists of the cross-correlation between the terms with the same noise matrix. For $\mathbf{N}_{t}$ only, there is one term with noise matrix $\mathbf{N}_{t}$, so

$$
\begin{aligned}
& \delta_{1}=\operatorname{trace}\left\{\operatorname{Re}\left(\mathbf{N}_{t} \mathbf{H}_{t-1}^{H} \mathbf{S}_{t-1}^{H} \mathbf{Q}_{t-1} \mathbf{Q}_{t}^{H}\right)\right\}, \\
& \delta_{2}=\operatorname{trace}\left\{\operatorname{Re}\left(\mathbf{N}_{t} \mathbf{H}_{t}^{H} \mathbf{S}_{t}^{H}\right)\right\},
\end{aligned}
$$

hence, the cross-correlation for $\mathbf{N}_{t}$ is

$$
\begin{aligned}
\Psi_{0}= & E\left[\delta_{1} \delta_{2}\right] \\
= & N_{0}\left(\sum_{\substack{i=1, \ldots, n_{R} \\
j=1, \ldots, n_{T}}}\left|h_{i j}\right|^{2}\right) \\
& \times \operatorname{trace}\left\{\operatorname{Re}\left(\mathbf{S}_{t} \mathbf{S}_{t-1}^{H} \mathbf{Q}_{t-1} \mathbf{Q}_{t}^{H}\right)\right\} \\
= & N_{0}\left(\sum_{\substack{i=1, \ldots, n_{R} \\
j=1, \ldots, n_{T}}}\left|h_{i j}\right|^{2}\right) \operatorname{trace}\left\{\operatorname{Re}\left(\mathbf{D}_{t}\right)\right\}
\end{aligned}
$$

For $\mathbf{N}_{t-1}$

$$
\begin{aligned}
\delta_{1} & =\operatorname{trace}\left\{\operatorname{Re}\left(\mathbf{S}_{t} \mathbf{H}_{t} \mathbf{N}_{t-1}^{H} \mathbf{Q}_{t-1} \mathbf{Q}_{t}^{H}\right)\right\} \\
& =\operatorname{trace}\left\{\operatorname{Re}\left(\mathbf{N}_{t} \mathbf{H}_{t}^{H} \mathbf{S}_{t}^{H} \mathbf{Q}_{t-1} \mathbf{Q}_{t}^{H}\right)\right\}, \\
\delta_{2} & =\operatorname{trace}\left\{\operatorname{Re}\left(\mathbf{H}_{t} \mathbf{N}_{t-1}^{H} \mathbf{S}_{t-1}\right)\right\} \\
& =\operatorname{trace}\left\{\operatorname{Re}\left(\mathbf{N}_{t-1} \mathbf{H}_{t}^{H} \mathbf{S}_{t-1}^{H}\right)\right\} .
\end{aligned}
$$

The cross-correlation for $\mathbf{N}_{t-1}$ is

$$
\begin{aligned}
\Psi_{1} & =E\left[\delta_{1} \delta_{2}\right] \\
& =N_{0}\left(\sum_{\substack{i=1, \ldots, n_{R} \\
j=1, \ldots, n_{T}}}\left|h_{i j}\right|^{2}\right) \times \operatorname{trace}\left\{\operatorname{Re}\left(\mathbf{S}_{t} \mathbf{S}_{t}^{H} \mathbf{Q}_{t} \mathbf{Q}_{t}^{H}\right)\right\} \\
& =N_{0}\left(\sum_{\substack{i=1, \ldots, n_{R} \\
j=1, \ldots, n_{T}}}\left|h_{i j}\right|^{2}\right) \operatorname{trace}\left\{\operatorname{Re}\left(\mathbf{D}_{t}\right)\right\}
\end{aligned}
$$

Therefore,

$$
\begin{aligned}
\Psi & =\Psi_{0}+\Psi_{1} \\
& =2 N_{0}\left(\sum_{\substack{i=1, \ldots, n_{R} \\
j=1, \ldots, n_{T}}}\left|h_{i j}\right|^{2}\right) \operatorname{trace}\left\{\operatorname{Re}\left(\mathbf{D}_{t}\right)\right\} .
\end{aligned}
$$

Finally, substituting Eqs. (29) and (30) into Eq. (26), we obtain Eq. (17).

\section{Acknowledgements}

This work was supported by the Vietnam National Foundation for Science and Technology Development under Grant 102.02-2015.23.

\section{Authors' contributions}

The authors have contributed jointly to all parts on the preparation of this manuscript, and all authors read and approved the final manuscript.

\section{Competing interests}

The authors declare that they have no competing interests.

\section{Publisher's Note}

Springer Nature remains neutral with regard to jurisdictional claims in published maps and institutional affiliations.

\section{Author details}

${ }^{1}$ Le Quy Don Technical University, Hoang Quoc Viet Street, Hanoi, Vietnam. ${ }^{2}$ Hanoi Department of Science and Technology, Hanoi, Vietnam. ${ }^{3}$ Middlesex University, London, UK.

Received: 21 July 2017 Accepted: 17 December 2017

Published online: 04 January 2018

\section{References}

1. R Mesleh, H Haas, C Ahn, S Yun, in First International Conference on Communications and Networking. Spatial modulation - a new low complexity spectral efficiency enhancing technique (IEEE, Beijing, 2006), pp. 1-5. http://ieeexplore.ieee.org/document/4149911/

2. R Mesleh, H Haas, S Sinanovic, CW Ahn, S Yun, Spatial modulation. IEEE Trans. Veh. Technol. 57(4), 2228-2241 (2008)

3. P Wolniansky, G Foschini, G Golden, Valenzuela R, in URSI International Symposium on Signals, Systems, and Electronics. Conference Proceedings. V-BLAST: an architecture for realizing very high data rates over the rich-scattering wireless channel (IEEE, Pisa, 1998), pp. 295-300. http:// ieeexplore.ieee.org/document/738086/

4. S Alamouti, A simple transmit diversity technique for wireless communications. IEEE J. Sel. Areas Commun. 16(8), 1451-1458 (1998)

5. V Tarokh, N Seshadri, A Calderbank, Space-time codes for high data rate wireless communication: performance criterion and code construction. IEEE Trans. Inform. Theory. 44(2), 744-765 (1998)

6. S Sugiura, S Chen, L Hanzo, Coherent and differential space-time shift keying: a dispersion matrix approach. IEEE Trans. Commun. 58(11), 3219-3230 (2010)

7. R Rajashekar, KVS Hari, L Hanzo, Structured dispersion matrices from division algebra codes for space-time shift keying. IEEE Signal Process. Lett. 20(4), 371-374 (2013)

8. R Rajashekar, KVS Hari, L Hanzo, in Global Telecommunications Conference (GLOBECOM 2011). Field extension code based dispersion matrices for coherently detected Space-Time Shift Keying. (IEEE, 2011), pp. 1-5. http:// ieeexplore.ieee.org/document/6134344/

9. R Rajashekar, KVS Hari, L Hanzo, Reduced-complexity ML detection and capacity-optimized training for spatial modulation systems. IEEE Trans. Commun. 62(1), 112-125 (2014)

10. H Men, M Jin, A Low-Complexity ML Detection Algorithm for Spatial Modulation Systems With $M$-PSK Constellation. IEEE Commun. Lett. 18(8), 1375-1378 (2014)

11. B Hassibi, BM Hochwald, How much training is needed in multiple-antenna wireless links. IEEE Trans. Inform. Theory. 49(4), 951-963 (2003)

12. F You, P Yang, Y Xiao, S Rong, D Ke, S Li, Blind Detection for Spatial Modulation Systems Based on Clustering. IEEE Commun. Lett. 21(11), 2392-2395 (2017)

13. $\vee$ Tarokh, $H$ Jafarkhani, A differential detection scheme for transmit diversity. IEEE J. Sel. Areas Commun. 18(7), 1169-1174 (2000)

14. SCheung, R Schober, Differential spatial multiplexing. IEEE Trans. Wirel. Commun. 5(8), 2127-2135 (2006)

15. Y Bian, M Wen, X Cheng, HV Poor, B Jiao, in Global Communications Conference (GLOBECOM). A differential scheme for spatial modulation (IEEE, 2013). http://ieeexplore.ieee.org/document/6831686/

16. Y Bian, X Cheng, M Wen, L Yang, HV Poor, B Jiao, Differential spatial modulation. IEEE Trans. Veh. Technol. 64(7), 3262-3268 (2015)

17. J Li, M Wen, X Cheng, Y Yan, S Song, MH Lee, Differential spatial modulation with Gray coded antenna activation order. IEEE Commun. Lett. 20(6), 1100-1103 (2016). http://ieeexplore.ieee.org/document/ 7458134/ 
18. R Rajashekar, N Ishikawa, S Sugiura, KVS Hari, L Hanzo, Full-diversity dispersion matrices from algebraic field extensions for differential spatial modulation. IEEE Trans. Veh. Technol. 66(1), 385-394 (2017). http:// ieeexplore.ieee.org/document/7422823/

19. M Zhang, M Wen, $X$ Cheng, L Yang, in Global Communications Conference (GLOBECOM), 2015 IEEE. Pre-coding aided differential spatial modulation (IEEE, 2015). http://ieeexplore.ieee.org/document/7417628/

20. MT Le, VD Ngo, HA Mai, XN Tran, in The 2012 International Conference on Advanced Technologies for Communications. High rate space time block coded spatial modulation (IEEE, Hanoi, 2012), pp. 278-282. http:// ieeexplore.ieee.org/document/6404276/

21. TP Nguyen, MT Le, VD Ngo, XN Tran, HW Choi, in 2014 IEEE 79th Vehicular Technology Conference (VTC Spring). Spatial modulation for high-rate transmission systems, (IEEE, Seoul, 2014). http://ieeexplore.ieee.org/ document/7023105/

22. H Jafarkhani, A quasi-orthogonal space-time block code. IEEE Trans. Commun. 49(1), 1-4 (2001)

23. TL Marzetta, BM Hochwald, Capacity of a mobile multiple-antenna communication link in Rayleigh flat fading. IEEE Trans. Inform. Theory. 45(1), 139-157 (1999)

24. J Yuan, Z Chen, B Vucetic, W Firmanto, Performance and design of space-time coding in fading channels. IEEE Trans. Commun. 51(12), 1991-1996 (2003)

25. MK Simon, M-S Alouini, Some new results for integrals involving the generalized Marcum Q function and their application to performance evaluation over fading channels. IEEE Trans. Wireless Commun. 2(3), 611-625 (2003)

26. JG Proakis, Digital Communications. (McGraw-Hill, New York, 1998)

27. D Divsalar, MK Simon, Multiple-symbol differential detection of MPSK. IEEE Trans. Commun. 38(3), 300-308 (1990)

28. Z Liu, GB Giannakis, BL Hughes, Double differential spacetime block coding for time-selective fading channels. IEEE Trans. Commun. 49(9), 1529-1536 (2001)

\section{Submit your manuscript to a SpringerOpen ${ }^{\circ}$ journal and benefit from:}

- Convenient online submission

- Rigorous peer review

- Open access: articles freely available online

- High visibility within the field

- Retaining the copyright to your article

Submit your next manuscript at $>$ springeropen.com 